\title{
Die Johannesapokalypse
}

Kontexte - Konzepte - Wirkungen

Hrsg. v. Jörg Frey, James A. Kelhoffer u. Franz Tóth

Die Johannesapokalypse Herausgegeben von JÖRG FREY,JAMES A. KELHOFFER und FRANZ TÓTH

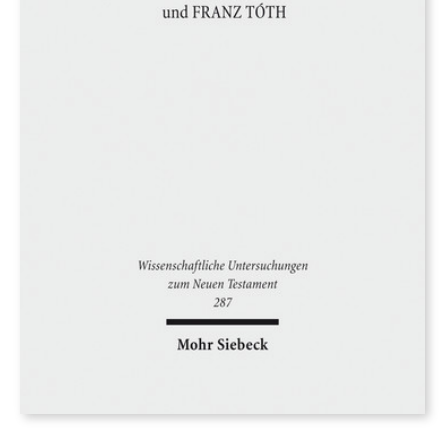

2012. XI, 867 Seiten. WUNT I 287

ISBN 978-3-16-152124-9 DOI 10.1628/978-3-16-152124-9 eBook PDF 204,00€ ISBN 978-3-16-150603-1 Leinen $204,00 €$
Die Johannesapokalypse ist zu einem Schwerpunkt der gegenwärtigen neutestamentlichen Forschung avanciert. Ihre historische Einordnung und Datierung sowie ihre traditions- und religionsgeschichtlichen Hintergründe werden ebenso intensiv diskutiert wie ihre literarische Rhetorik und ihre theologische Konzeption. Der Band vereint Vorträge eines Münchener Symposiums mit zusätzlich angefragten Beiträgen. Im ersten Teil »Kontexte« werden Fragen der Textgestalt und der Traditionsbezüge, der historischen Einordnung und der Datierung erörtert. Ein zweiter Teil thematisiert »Konzepte« der Apokalypse; darin wird u.a. Fragen nach der Christologie und Eschatologie nachgegangen, ferner stehen die Rede vom Martyrium und Untersuchungen zur Redaktion und theologischen Intention des Werkes im Mittelpunkt. Der abschließende dritte Teil »Wirkungen« fokussiert Schwerpunkte der Rezeption vom Montanismus und der Thomasapokalypse über Laktanz und Andreas von Caesarea bis zur orthodoxen Liturgie und zur Kunst William Blakes. Die Beiträge bieten einen repräsentativen Einblick in die wesentlichen Diskurse zur gegenwärtigen Apokalypseforschung.

\section{Inhaltsübersicht}

Franz Tóth: Erträge und Tendenzen der gegenwärtigen Forschung zur Johannesapokalypse I. Kontexte

Martin Karrer: Der Text der Johannesapokalypse - Thomas Witulski: Der römische Kaiser Hadrian und die neutestamentliche Johannesapokalypse - Stefan Witetschek: Ein weit geöffnetes Zeitfenster? Überlegungen zur Datierung der Johannesapokalypse - Michael Labahn: Die Septuaginta und die Johannesapokalypse: Möglichkeiten und Grenzen einer Verhältnisbestimmung im Spiegel von kreativer Intertextualität und Textentwicklungen - Loren T. Stuckenbruck /Mark D. Mathews: The Apocalypse of John, 1 Enoch, and the Question of Influence - Enno Edzard Popkes: Vollendete Gottesgegenwart: Anmerkungen zu den traditionsgeschichtlichen Bezugsgrößen von Apk 21,1-5 - Clare K. Rothschild: Principle, Power, and Purgation in the Letter to the Church in Laodicea (Rev 3:14-22) - Jan Dochhorn: Beliar als Endtyrann in der Ascensio Isaiae. Ein Beitrag zur Eschatologie und Satanologie des frühen Christentums sowie zur Erforschung der Apokalypse des Johannes

\section{Konzepte}

Franz Tóth: Von der Vision zur Redaktion. Untersuchungen zur Komposition, Redaktion und Intention der Johannesapokalypse - Hans-Georg Gradl: Buch und Brief. Zur motivischen, literarischen und kommunikativen Interdependenz zweier medialer Typen in der Johannes-Offenbarung - Konrad Huber: Jesus Christus - der Erste und der Letzte. Zur Christologie der Johannesapokalypse - Jörg Frey: Was erwartet die Johannesapokalypse? Zur Eschatologie des letzten Buchs der Bibel - James A. Kelhoffer: The Relevance of Revelation's Date and the Imperial Cult for John's Appraisal of the Value of Christians' Suffering in Revelation 1-3 - Jan Willem van Henten: The Concept of Martyrdom in Revelation - Roland Bergmeier: Zeugnis und Martyrium

\section{Rezeption}

William Tabbernee: The Appearance of the New Jerusalem in Montanist Interpretation and the Revelation of John - Tobias Nicklas: Die apokryphe Thomasapokalypse und ihre Rezeption der Offenbarung desJohannes - Valentin Fàbrega: Laktanz und die Apokalypse - Juan Hernández, Jr.: Andrew of Caesarea and His Reading of Revelation. Catechesis and Paranesis Konstantin Nikolakopoulos: Die Apokalypse des Johannes und die orthodoxe Liturgie. Anknüpfungspunkte zwischen Apokalypse und orthodoxem Kultus - Christopher Rowland: 'Mr. Blake: apo- or rather ana-calyptic Poet and Painter': Apocalyptic Hermeneutics in Action

Jörg Frey Geboren 1962; 1996 Promotion; 1998 Habilitation; Professur für neutestamentliche Wissenschaft mit den Schwerpunkten Antikes Judentum und Hermeneutik an der Theologischen Fakultät der Universität Zürich und Research Associate der University of the Free State, Bloemfontein/ZA. https://orcid.org/0000-0001-6628-8834

James A. Kelhoffer Born 1970; 1999 PhD University of Chicago; 2003 postdoctoral fellow Catholic Biblical Association of America; 2007 postdoctoral fellow Alexander von Humboldt Foundation at Ludwig-Maximilians-Universität München; currently Professor of New Testament Studies at Uppsala University (Sweden).

Franz Tóth Geboren 1972; 1993-99 Studium der Philosophie und Theologie; 2005 Promotion; 2014 Habilitation; 2015 Lehrstuhlvertretung für Prof. Dr. Matthias Konradt an der Ruprecht-Karls-Universität Heidelberg; seit 2017 Privatdozent an der Theologischen Fakultät der Universität Zürich.

Jetzt bestellen:

https://mohrsiebeck.com/buch/die-johannesapokalypse-9783161521249?no_cache=1

order@mohrsiebeck.com

Telefon: +49 (0)7071-923-17

Telefax: $+49(0) 7071-51104$ 